\title{
Article
}

\section{Tocilizumab Improves the Prognosis of COVID-19 in Patients with High IL-6}

\author{
Robert Flisiak ${ }^{1, *}$, Jerzy Jaroszewicz ${ }^{2}$, Magdalena Rogalska ${ }^{1}$, Tadeusz Łapiński ${ }^{1}$, \\ Aleksandra Berkan-Kawińska ${ }^{3}{ }^{(\mathbb{B}}$, Beata Bolewska ${ }^{4}$, Magdalena Tudrujek-Zdunek ${ }^{5}{ }^{\circledR}$, Dorota Kozielewicz ${ }^{6}$, \\ Marta Rorat ${ }^{7,8} \mathbb{D}^{\text {, Piotr Leszczyński }}{ }^{9,10}$, Krzysztof Kłos ${ }^{11}{ }^{\mathbb{D}}$, Justyna Kowalska ${ }^{12}$, Paweł Pabjan ${ }^{13}$, \\ Anna Piekarska ${ }^{3}$, Iwona Mozer-Lisewska ${ }^{4}$, Krzysztof Tomasiewicz ${ }^{5}$, Małgorzata Pawłowska ${ }^{6}$, \\ Krzysztof Simon ${ }^{14}$, Joanna Polanska ${ }^{15}$ (b) and Dorota Zarębska-Michaluk ${ }^{13}[$
}

check for

updates

Citation: Flisiak, R.; Jaroszewicz, J.; Rogalska, M.; Łapiński, T.; BerkanKawińska, A.; Bolewska, B.; TudrujekZdunek, M.; Kozielewicz, D.; Rorat, M.; Leszczyński, P.; et al. Tocilizumab Improves the Prognosis of COVID-19 in Patients with High IL-6. J. Clin. Med. 2021, 10, 1583. https://doi.org/ $10.3390 /$ jcm 10081583

Academic Editor: Marco Sebastiani

Received: 6 March 2021

Accepted: 5 April 2021

Published: 9 April 2021

Publisher's Note: MDPI stays neutral with regard to jurisdictional claims in published maps and institutional affiliations.

Copyright: (c) 2021 by the authors. Licensee MDPI, Basel, Switzerland. This article is an open access article distributed under the terms and conditions of the Creative Commons Attribution (CC BY) license (https:// creativecommons.org/licenses/by/ $4.0 /)$.
1 Department of Infectious Diseases and Hepatology, Medical University of Białystok, 15-089 Białystok, Poland; pmagdar@gmail.com (M.R.); twlapinski@gmail.com (T.Ł.)

2 Department of Infectious Diseases and Hepatology, Medical University of Silesia, 40-055 Katowice, Poland; jerzy.jr@gmail.com

3 Department of Infectious Diseases and Hepatology, Medical University of Łódź, 90-549 Łódź, Poland; aleksandra.berkan@gmail.com (A.B.-K.); annapiekar@gmail.com (A.P.)

4 Department of Infectious Diseases, University of Medical Sciences, 61-701 Poznań, Poland; bbolewska@ump.edu.pl (B.B.); iwonalisewska@poczta.onet.pl (I.M.-L.)

5 Department of Infectious Diseases and Hepatology, Medical University of Lublin, 20-059 Lublin, Poland; magdalena.tudrujek@gmail.com (M.T.-Z.); tomaskdr@poczta.fm (K.T.)

6 Department of Infectious Diseases and Hepatology, Faculty of Medicine, Collegium Medicum in Bydgoszcz, Nicolaus Copernicus University, 87-100 Torun, Poland; d.kozielewicz@wsoz.pl (D.K.); mpawlowska@cm.umk.pl (M.P.)

7 Department of Forensic Medicine, Wrocław Medical University, 50-367 Wrocław, Poland; marta.rorat@gmail.com

8 First Infectious Diseases Ward, Gromkowski Regional Specialist Hospital in Wrocław, 51-149 Wrocław, Poland 9 Department of Rheumatology, Rehabilitation and Internal Medicine, Poznan University of Medical Sciences, 61-701 Poznań, Poland; piotr_leszczynski@wp.pl

10 Department of Rheumatology and Osteoporosis, Szpital im. J. Strusia w Poznaniu, Szpital im. J. Strusia, 61-285 Poznań, Poland

11 Department of Infectious Diseases and Allergology, Military Institute of Medicine, 04-141 Warsaw, Poland; kklos@wim.mil.pl

12 Department of Adults' Infectious Diseases, Medical University of Warsaw, 02-091 Warsaw, Poland; jdkowalska@gmail.com

13 Department of Infectious Diseases, Jan Kochanowski University, 25-369 Kielce, Poland; pabjan3@tlen.pl (P.P.); dorota1010@tlen.pl (D.Z.-M.)

14 Department of Infectious Diseases and Hepatology, Wrocław Medical University, 50-367 Wrocław, Poland; krzysimon@gmail.com

15 Department of Data Science and Engineering, Silesian University of Technology, 44-100 Gliwice, Poland; Joanna.Polanska@polsl.pl

* Correspondence: robert.flisiak1@gmail.com

Abstract: Despite direct viral effect, the pathogenesis of coronavirus disease 2019 (COVID-19) includes an overproduction of cytokines including interleukin 6 (IL-6). Therefore, tocilizumab (TOC), a monoclonal antibody against IL-6 receptors, was considered as a possible therapeutic option. Patients were selected from the SARSTer database, containing 2332 individuals with COVID-19. Current study included 825 adult patients with moderate to severe course. Analysis was performed in 170 patients treated with TOC and 655 with an alternative medication. The end-points of treatment effectiveness were death rate, need for mechanical ventilation, and clinical improvement. Patients treated with TOC were balanced compared to non-TOC regarding gender, age, BMI, and prevalence of coexisting conditions. Significant effect of TOC on death was demonstrated in patients with baseline IL-6 > $100 \mathrm{pg} / \mathrm{mL}$ (hazard ratio [HR]: 0.21, 95\% confidence interval [CI]: 0.08-0.57). The best effectiveness of TOC was achieved in patients with a combination of baseline IL- $6>100 \mathrm{pg} / \mathrm{mL}$ and either $\mathrm{SpO} 2 \leq 90 \%$ (HR: 0.07 ) or requiring oxygen supplementation (HR: 0.18). Tocilizumab administration in COVID-19 reduces mortality and speeds up clinical improvement in patients with 
a baseline concentration of IL- $6>100 \mathrm{pg} / \mathrm{mL}$, particularly if they need oxygen supplementation owing to the lower value of $\mathrm{SpO} 2 \leq 90 \%$.

Keywords: COVID-19; SARS-CoV-2; interleukin-6; tocilizumab; therapy

\section{Introduction}

A novel coronavirus named severe acute respiratory syndrome coronavirus 2 (SARSCoV-2) was identified in December 2019 and found to be responsible for an outbreak of respiratory tract infections discovered in Wuhan, China. The outbreak of the disease known as a coronavirus disease 2019 (COVID-19) was announced as a global pandemic by the World Health Organization (WHO) in March 2020. The search for effective therapy focused on repurposing of approved drugs with confirmed activity against other viruses, that included, for example, remdesivir (RDV), which was previously studied for the treatment of Ebola virus disease as well as SARS-CoV-1 and middle east respiratory syndrome (MERS) coronaviruses [1,2]. Based on findings from phase III clinical trials and real-world experience study, RDV received both American and European authorization [3-5]. Recommendations were also given to low-molecular-weight heparin and dexamethasone [6,7]. However, the pathogenesis of COVID-19 is complicated and includes, in addition to direct viral effect and coagulopathy, an overproduction of proinflammatory cytokines termed a cytokine storm, which is responsible for organ damage and is considered a major reason for death due to COVID-19 [8]. Unfortunately, standard anti-inflammatory treatments appear to be insufficient for controlling the cytokine storm. Concentrations of several proinflammatory cytokines, including interleukin (IL)-6, are substantially increased in patients with severe COVID-19 [9]. Higher concentrations of IL-6 was shown to be associated with faster progression of the disease and worse prognosis. Therefore, tocilizumab (TOC), an inhibitor of the IL-6 receptors, was considered as a possible therapeutic option [9-11]. Data from several studies have been contradictory mostly because of the difficulties in the selection of optimal population and finding the proper stage of the disease for administration [12-14]. Although the most recent randomized, double-blind, placebo-controlled trial by Stone et al. was not able to confirm the effectiveness of TOC, authors did not exclude the possible benefit from interleukin- 6 receptor blockade in some patient populations because of wide confidence intervals for efficacy comparisons [14].

The purpose of the study is to search for the population of patients with severe COVID-19, which could obtain maximal benefit from the administration of tocilizumab, and identify the predictors of response to the treatment with this drug.

\section{Materials and Methods}

Patients were selected from the SARSTer national database, which included 2332 patients treated between 1 March and 31 October 2020 in 30 Polish centers. This ongoing project, supported by the Polish Association of Epidemiologists and Infectiologists, is a national real-world experience study assessing treatment in patients with COVID-19. The decision about the treatment regimen was taken entirely by the treating physician concerning current knowledge and recommendations of the Polish Association of Epidemiologists and Infectiologists [15-17]. The SARSTer study was approved by the Ethical Committee of the Medical University of Białystok. If necessary, the local bioethics committees approved experimental use of drugs in patients with COVID-19. Patients aged below 18 years, those with oxygen saturation $>95 \%$, or acute respiratory distress syndrome (ARDS) at baseline were excluded from the database of 2332 patients. As a result, the current study included 825 adult patients with moderate to severe course of the disease.

Among those 825 patients, the retrospective analysis was carried out in 170 patients treated with tocilizumab (RoActemra, Roche Pharma AG) and 655 patients who did not receive this medication as well as any other monoclonal antibody directed against cytokine 
receptors. Tocilizumab was administered intravenously at $8 \mathrm{mg} / \mathrm{kg}$ (maximum dose: $800 \mathrm{mg}$ ) in a single dose (1-h infusion) after exclusion of severe bacterial and HBV infection. If no improvement was observed, the second dose was considered after 8 to $12 \mathrm{~h}$ (administered in $42 \%$ patients) according to the national recommendations [15-17]. Data were entered retrospectively and submitted online by a web-based platform operated by Tiba sp. z o.o. Parameters collected at baseline included age, gender, body mass index (BMI), coexisting conditions, other medication-related to COVID-19, clinical status at admission, and adverse events. Baseline clinical status at hospital admission was classified according to oxygen saturation ( $\mathrm{SpO} 2$ ) $91-95 \%$, or $\mathrm{SpO} 2 \leq 90 \%$, as well as based on the score on an ordinal scale.

The end-points of treatment effectiveness were rate of death, need for mechanical ventilation, and clinical improvement in the ordinal scale based on WHO recommendations modified to fit the specificity of the national health care system. Clinical improvement was defined as at least a 2-point decrease from baseline to 14, 21, and 28 days of hospitalization. The ordinal scale was scored as follows: (1) unhospitalized, no activity restrictions; (2) unhospitalized, no activity restrictions and/or requiring oxygen supplementation at home; (3) hospitalized, does not require oxygen supplementation and does not require medical care; (4) hospitalized, requiring no oxygen supplementation, but requiring medical care; (5) hospitalized, requiring normal oxygen supplementation; (6) hospitalized, on non-invasive ventilation with high-flow oxygen equipment; (7) hospitalized, for invasive mechanical ventilation or extracorporeal membrane oxygenation (ECMO); (8) death.

To identify possible predictors of response to the treatment with TOC, we compared rates of achieved end-points in patients receiving versus not receiving TOC. The following baseline predictors were included: age above 70 years, the need for oxygen high flow (ordinal scale 6 points) at baseline, clinical worsening during 7 days of hospitalization in patients with regular oxygen supplementation at baseline (5 points in original scale), $\mathrm{SpO} 2<90 \%$ at baseline, and several laboratory measures at baseline, such as IL-6 > $100 \mathrm{pg} / \mathrm{mL}$, C-reactive protein (CRP) > $200 \mathrm{mg} / \mathrm{L}$, neutrophils > 7500/ $\mu \mathrm{L}$, lymphocytes $>1200 / \mu \mathrm{L}$, D-dimer $>1000 \mu \mathrm{g} / \mathrm{L}$, and procalcitonin $>0.1 \mathrm{ng} / \mathrm{mL}$.

\section{Statistical Analysis}

The results are expressed as mean \pm standard deviation (SD) or $n(\%)$. $p$ values of $<0.05$ were considered to be statistically significant. The significance of difference was calculated by Fisher's exact test for nominal variables and by Mann-Whitney U and Kruskal Wallis ANOVA for continuous and ordinal variables. Due to the highly variable group size, the Fisher's p-values were accompanied by odds ratio (OR) as the effect size measure independent of the sample size. The association between variables was measured by Spearman's rank correlation coefficient and its significance test $p$-values. Survival analyses were performed by log-rank (Mantel-Cox) test supported by the Mantel-Haenszel hazard ratio (MH HR) and its 95\% confidence interval as the effect size measure and depicted as Kaplan-Meier (KM) plots. The threshold value of IL-6, splitting between the low and the high IL-6 level groups, was found as maximizing the non-TOC vs. TOC hazard ratio value in the high IL-6 group providing the significant differences between the IL-6 group-specific KM survival functions (as measured by log-rank test $p$-value). Univariable comparisons were calculated by GraphPad Prism 5.1 (GraphPad Software, Inc., La Jolla, CA, USA).

\section{Results}

Among 825 patients included in the study, 170 received therapy with TOC and 655 did not receive TOC. As shown in Table 1, groups were balanced based on gender, age, and BMI, but there was a predominance of males in both arms. Patients treated with TOC more frequently demonstrated a course of the disease with $\mathrm{SpO} 2 \leq 90 \%$ at admission to the hospital $(65.9 \%)$ compared to those without TOC (37.7\%). Moreover, patients treated with TOC more often required normal or high flow oxygen supplementation $(93.6 \%)$ compared to the non-TOC group $(76.8 \%)$. The prevalence of coexisting conditions was similar in both 
groups, but patients treated with TOC more frequently received other medications related to COVID-19 (Table 1).

Table 1. Baseline demographic and clinical characteristics of included patients.

\begin{tabular}{|c|c|c|c|c|}
\hline Characteristic & $\begin{array}{l}\text { All Patients } \\
\quad n=825\end{array}$ & $\begin{array}{l}\text { Tocilizumab } \\
\quad n=170\end{array}$ & $\begin{array}{l}\text { No Tocilizumab } \\
\quad n=655\end{array}$ & $p$ \\
\hline \multicolumn{5}{|l|}{ Age } \\
\hline Mean (SD) & $63.1(15.1)$ & $63.2(13.8)$ & $63.0(15.4)$ & 0.94 \\
\hline$>70$ years $(\%)$ & $267(32.4)$ & $53(31.2)$ & $214(32.7)$ & 0.78 \\
\hline \multicolumn{5}{|l|}{ Gender } \\
\hline Female, $n(\%)$ & $337(40.8)$ & $60(35.3)$ & $277(42.3)$ & 0.11 \\
\hline Male, $n(\%)$ & $488(59.2)$ & $110(64.7)$ & $378(57.7)$ & 0.11 \\
\hline Body mas index, mean (SD) & $28.8(4.9)$ & $29.7(4.8)$ & $28.5(5.0)$ & 0.01 \\
\hline \multicolumn{5}{|l|}{ Disease severity at the baseline, $n(\%)$} \\
\hline Oxygen saturation $91-95 \%$ & 466 (56.5) & $58(34.1)$ & $408(62.3)$ & $<0.001$ \\
\hline Oxygen saturation $\leq 90 \%$ & $359(43.5)$ & $112(65.9)$ & $247(37.7)$ & $<0.001$ \\
\hline \multicolumn{5}{|l|}{ Score on ordinal scale, $n(\%)$} \\
\hline $\begin{array}{l}\text { 4. Hospitalized, requiring no oxygen supplementation, but } \\
\text { requiring medical care }\end{array}$ & $163(19.8)$ & $11(6.5)$ & $152(23.2)$ & $<0.001$ \\
\hline 5. Hospitalized, requiring normal oxygen supplementation & 615 (74.5) & 147 (86.5) & $468(71.5)$ & $<0.001$ \\
\hline $\begin{array}{l}\text { 6. Hospitalized, on non-invasive ventilation with high-flow } \\
\text { oxygen equipment }\end{array}$ & $47(5.7)$ & $12(7.1)$ & $35(5.3)$ & 0.36 \\
\hline Coexisting conditions, $n(\%)$ & $638(77.3)$ & $131(77.1)$ & $507(77.4)$ & 0.91 \\
\hline \multicolumn{5}{|c|}{ Other medications related to COVID-19, $n(\%)$} \\
\hline Remdesivir & $284(34.4)$ & $67(39.4)$ & $217(33.1)$ & 0.15 \\
\hline Dexamethason & $272(33.0)$ & $83(48.8)$ & $189(28.9)$ & $<0.001$ \\
\hline Covalescent plasma & $103(12.5)$ & 29 (17.1) & $74(11.3)$ & 0.05 \\
\hline Low molecular weight heparin & $713(86.5)$ & $170(100.0)$ & $543(82.9)$ & $<0.001$ \\
\hline
\end{tabular}

As shown in Table 2, the rate of clinical improvement after 21 and 28 days was significantly better in patients who did not receive TOC. However, a statistically significant effect of TOC on rates of death was demonstrated in patients with baseline IL-6 exceeding $100 \mathrm{pg} / \mathrm{mL}$ or those needing oxygen supplementation at baseline whose condition worsened within the initial 7 days of hospitalization (Table 2, Figure 1). As shown with the Kaplan-Meier analysis, there were no significant differences between TOC and nonTOC arms when the analysis was carried out in all patients or those with baseline IL-6 concentration below $100 \mathrm{pg} / \mathrm{mL}$. Further analysis included the correlation between IL6 concentration and several possible clinical and laboratory indices associated with the course of the disease. Among patients with baseline $\mathrm{SpO} 2 \leq 90 \%$, who are potential TOC recipients, significant correlation was demonstrated among serum concentrations of IL-6 and $\mathrm{SpO} 2$, levels of C-reactive protein, procalcitonin D-dimers, as well as white blood cell and neutrophil counts (Table 3).

Table 2. Tocilizumab effect on rates of death, need for mechanical ventilation, and clinical improvement depending on possible outcomes predictors.

\begin{tabular}{ccccc}
\hline Outcomes & Tocilizumab & No Tocilizumab & $p$ & OR \\
(95\%CI) & & & \\
\hline$n$ & Overall & & \\
\hline Death, $n(\%)$ & 170 & 655 & & \\
\hline Mechanical ventillation, $n(\%)$ & $19(11.2)$ & $70(10.7)$ & 0.89 & $(0.61-1.80)$ \\
\hline
\end{tabular}


Table 2. Cont.

\begin{tabular}{|c|c|c|c|c|}
\hline Outcomes & Tocilizumab & No Tocilizumab & $p$ & $\begin{array}{c}\text { OR } \\
(95 \% \mathrm{CI})\end{array}$ \\
\hline \multicolumn{5}{|c|}{ Overall } \\
\hline Clinical improvement after 14 days, $n(\%)$ & $70(41.2)$ & $351(53.6)$ & 0.004 & $\begin{array}{c}0.61 \\
(0.43-0.85)\end{array}$ \\
\hline Clinical improvement after 21 days, $n(\%)$ & $112(65.9)$ & $492(75.1)$ & 0.02 & $\begin{array}{c}0.64 \\
(0.44-0.92)\end{array}$ \\
\hline Clinical improvement after 28 days, $n(\%)$ & $134(78.8)$ & $531(81.1)$ & 0.51 & $\begin{array}{c}0.87 \\
(0.57-1.32)\end{array}$ \\
\hline \multicolumn{5}{|c|}{ Age $>70$ years } \\
\hline$n$ & 53 & 215 & & \\
\hline Death, $n(\%)$ & $11(20.8)$ & $49(22.8)$ & 0.85 & $\begin{array}{c}0.89 \\
(0.42-1.85)\end{array}$ \\
\hline Mechanical ventillation, $n(\%)$ & $5(9.4)$ & $20(9.3)$ & 1.00 & $\begin{array}{c}1.02 \\
(0.36-2.84)\end{array}$ \\
\hline Clinical improvement after 14 days, $n(\%)$ & $16(30.2)$ & $77(35.8)$ & 0.52 & $\begin{array}{c}0.83 \\
(0.43-1.59)\end{array}$ \\
\hline Clinical improvement after 21 days, $n(\%)$ & $28(52.8)$ & $124(57.7)$ & 0.54 & $\begin{array}{c}0.82 \\
(0.45-1.50)\end{array}$ \\
\hline Clinical improvement after 28 days, $n(\%)$ & $35(66.0)$ & $141(65.6)$ & 1.00 & $\begin{array}{c}1.02 \\
(0.54-1.92)\end{array}$ \\
\hline \multicolumn{5}{|c|}{ The need for oxygen high flow (score 6 in ordinal scale) at baseline } \\
\hline$n$ & 14 & 35 & & \\
\hline Death, $n(\%)$ & $4(28.6)$ & $12(34.3)$ & 1.00 & $\begin{array}{c}0.77 \\
(0.20-2.97)\end{array}$ \\
\hline Mechanical ventillation, $n(\%)$ & $4(28.6)$ & $8(22.9)$ & 0.72 & $\begin{array}{c}1.35 \\
(0.33-5.50)\end{array}$ \\
\hline Clinical improvement after 14 days, $n(\%)$ & $6(42.9)$ & $9(25.7)$ & 0.31 & $\begin{array}{c}2.17 \\
(0.59-7.97)\end{array}$ \\
\hline Clinical improvement after 21 days, $n(\%)$ & $9(64.3)$ & $15(42.9)$ & 0.21 & $\begin{array}{c}2.40 \\
(0.67-8.65)\end{array}$ \\
\hline Clinical improvement after 28 days, $n(\%)$ & $9(64.3)$ & $17(48.6)$ & 0.36 & $\begin{array}{c}1.91 \\
(0.53-6.85)\end{array}$ \\
\hline
\end{tabular}

Clinical worsening during 7 days of hospitalization in patients with regular oxygen supplementation at baseline (5 points in original scale)

\begin{tabular}{ccccc}
\hline$n$ & 41 & 55 & & \\
\hline Death, $n(\%)$ & $18(43.9)$ & $37(67.3)$ & $\mathbf{0 . 0 4}$ & $\mathbf{0 . 3 8}$ \\
\hline Mechanical ventillation, $n(\%)$ & $20(48.8)$ & $23(41.8)$ & 0.54 & 1.32 \\
\hline Clinical improvement after 14 days, $n(\%)$ & 0 & $2(3.6)$ & 0.51 & - \\
\hline Clinical improvement after 21 days, $n(\%)$ & $1(2.4)$ & $8(14.5)$ & 0.07 & $(0.01-1.23)$ \\
\hline Clinical improvement after 28 days, $n(\%)$ & $14(34.1)$ & $12(21.8)$ & 0.24 & $(0.75-4.61)$ \\
\hline
\end{tabular}


Table 2. Cont.

\begin{tabular}{|c|c|c|c|c|}
\hline Outcomes & Tocilizumab & No Tocilizumab & $p$ & $\begin{array}{c}\text { OR } \\
(95 \% \mathrm{CI})\end{array}$ \\
\hline \multicolumn{5}{|c|}{$\mathrm{SpO} 2 \leq 90 \%$ at the baseline } \\
\hline$n$ & 125 & 247 & & \\
\hline Death, $n(\%)$ & $23(17.6)$ & $52(21.1)$ & 0.59 & $\begin{array}{c}0.84 \\
(0.49-1.46)\end{array}$ \\
\hline Mechanical ventillation, $n(\%)$ & $23(17.6)$ & $30(11.7)$ & 0.12 & $\begin{array}{c}1.63 \\
(0.90-2.95)\end{array}$ \\
\hline Clinical improvement after 14 days, $n(\%)$ & $41(32.8)$ & $106(42.5)$ & 0.07 & $\begin{array}{c}0.65 \\
(0.41-1.10)\end{array}$ \\
\hline Clinical improvement after 21 days, $n(\%)$ & $66(52.8)$ & $156(62.8)$ & 0.06 & $\begin{array}{c}0.65 \\
(0.42-1.01)\end{array}$ \\
\hline Clinical improvement after 28 days, $n(\%)$ & $83(66.4)$ & $173(69.6)$ & 0.47 & $\begin{array}{c}0.84 \\
(0.53-1.34)\end{array}$ \\
\hline \multicolumn{5}{|c|}{ IL-6 $>100 \mathrm{pg} / \mathrm{mL}$ at baseline } \\
\hline$n$ & 56 & 42 & & \\
\hline Death, $n(\%)$ & $6(10.7)$ & $13(31.0)$ & 0.02 & $\begin{array}{c}0.27 \\
(0.10-0.78)\end{array}$ \\
\hline Mechanical ventillation, $n(\%)$ & $7(12.5)$ & $7(16.7)$ & 0.57 & $\begin{array}{c}0.71 \\
(0.23-2.20)\end{array}$ \\
\hline Clinical improvement after 14 days, $n(\%)$ & $22(39.3)$ & $14(33.3)$ & 0.67 & $\begin{array}{c}1.29 \\
(0.56-2.99)\end{array}$ \\
\hline Clinical improvement after 21 days, $n(\%)$ & $35(62.5)$ & $19(45.2)$ & 0.10 & $\begin{array}{c}2.02 \\
(0.89-4.55)\end{array}$ \\
\hline Clinical improvement after 28 days, $n(\%)$ & $41(73.2)$ & $23(54.8)$ & 0.08 & $\begin{array}{c}2.26 \\
(0.97-5.27)\end{array}$ \\
\hline \multicolumn{5}{|c|}{$\mathrm{CRP}>200 \mathrm{mg} / \mathrm{L}$ at the baseline } \\
\hline$n$ & 39 & 61 & & \\
\hline Death, $n(\%)$ & $6(15.4)$ & $13(21.3)$ & 0.60 & $\begin{array}{c}0.74 \\
(0.25-2.17)\end{array}$ \\
\hline Mechanical ventillation, $n(\%)$ & $6(15.4)$ & $10(16.4)$ & 1.00 & $\begin{array}{c}0.92 \\
(0.31-2.79)\end{array}$ \\
\hline Clinical improvement after 14 days, $n(\%)$ & $12(30.8)$ & $17(27.9)$ & 0.82 & $\begin{array}{c}1.15 \\
(0.48-2.78)\end{array}$ \\
\hline Clinical improvement after 21 days, $n(\%)$ & $20(51.3)$ & $35(57.4)$ & 0.68 & $\begin{array}{c}0.78 \\
(0.35-1.75)\end{array}$ \\
\hline Clinical improvement after 28 days, $n(\%)$ & $27(69.2)$ & $39(63.9)$ & 0.67 & $\begin{array}{c}1.27 \\
(0.54-2.99)\end{array}$ \\
\hline \multicolumn{5}{|c|}{ Neutrophils $>7500 / \mu \mathrm{L}$ at the baseline } \\
\hline$n$ & 39 & 90 & & \\
\hline Death, $n(\%)$ & $7(17.9)$ & $23(25.6)$ & 0.49 & $\begin{array}{c}0.63 \\
(0.25-1.64)\end{array}$ \\
\hline Mechanical ventillation, $n(\%)$ & $2(5.1)$ & $8(8.9)$ & 1.00 & $\begin{array}{c}0.55 \\
(0.11-2.73)\end{array}$ \\
\hline Clinical improvement after 14 days, $n(\%)$ & $15(38.5)$ & $33(36.7)$ & 0.84 & $\begin{array}{c}1.08 \\
(0.50-2.34)\end{array}$ \\
\hline Clinical improvement after 21 days, $n(\%)$ & $23(59.0)$ & $51(56.7)$ & 0.84 & $\begin{array}{c}1.10 \\
(0.51-2.35)\end{array}$ \\
\hline Clinical improvement after 28 days, $n(\%)$ & $28(71.8)$ & $59(65.6)$ & 0.54 & $\begin{array}{c}1.34 \\
(0.59-3.04)\end{array}$ \\
\hline
\end{tabular}


Table 2. Cont.

\begin{tabular}{|c|c|c|c|c|}
\hline Outcomes & Tocilizumab & No Tocilizumab & $p$ & $\begin{array}{c}\text { OR } \\
(95 \% \mathrm{CI})\end{array}$ \\
\hline \multicolumn{5}{|c|}{ Lymphocytes $>1200 / \mu \mathrm{L}$ at the baseline } \\
\hline$n$ & 47 & 239 & & \\
\hline Death, $n(\%)$ & $1(2.1)$ & $20(8.4)$ & 0.22 & $\begin{array}{c}0.24 \\
(0.03-1.82)\end{array}$ \\
\hline Mechanical ventillation, $n(\%)$ & 0 & $8(3.3)$ & 0.36 & - \\
\hline Clinical improvement after 14 days, $n(\%)$ & $28(59.6)$ & $142(59.4)$ & 1.00 & $\begin{array}{c}1.01 \\
(0.53-1.90)\end{array}$ \\
\hline Clinical improvement after 21 days, $n(\%)$ & $36(76.6)$ & $192(80.3)$ & 0.55 & $\begin{array}{c}0.80 \\
(0.38-1.69)\end{array}$ \\
\hline Clinical improvement after 28 days, $n(\%)$ & $43(91.5)$ & $201(84.1)$ & 0.26 & $\begin{array}{c}2.03 \\
(0.69-6.00)\end{array}$ \\
\hline \multicolumn{5}{|c|}{ D-dimers $>1000 \mu \mathrm{g} / \mathrm{L}$ at the baseline } \\
\hline$n$ & 75 & 221 & & \\
\hline Death, $n(\%)$ & $12(16.0)$ & $45(20.4)$ & 0.50 & $\begin{array}{c}0.74 \\
(0.37-1.50)\end{array}$ \\
\hline Mechanical ventillation, $n(\%)$ & $7(9.3)$ & $21(9.5)$ & 1.00 & $\begin{array}{c}0.98 \\
(0.40-2.41)\end{array}$ \\
\hline Clinical improvement after 14 days, $n(\%)$ & $25(33.3)$ & $101(45.7)$ & 0.08 & $\begin{array}{c}0.59 \\
(0.34-1.03)\end{array}$ \\
\hline Clinical improvement after 21 days, $n(\%)$ & $43(57.3)$ & $143(64.7)$ & 0.27 & $\begin{array}{c}0.73 \\
(0.43-1.25)\end{array}$ \\
\hline Clinical improvement after 28 days, $n(\%)$ & $54(72.0)$ & $158(71.5)$ & 1.00 & $\begin{array}{c}1.02 \\
(0.57-1.84)\end{array}$ \\
\hline \multicolumn{5}{|c|}{ Procalcitonin $>0.1 \mathrm{ng} / \mathrm{mL}$ at the baseline } \\
\hline$n$ & 92 & 193 & & \\
\hline Death, $n(\%)$ & $18(19.6)$ & $44(22.8)$ & 0.64 & $\begin{array}{c}0.82 \\
(0.44-1.52)\end{array}$ \\
\hline Mechanical ventillation, $n(\%)$ & $10(10.9)$ & $25(13.0)$ & 0.70 & $\begin{array}{c}0.82 \\
(0.37-1.79)\end{array}$ \\
\hline Clinical improvement after 14 days, $n(\%)$ & $34(37.0)$ & $74(38.3)$ & 0.89 & $\begin{array}{c}0.94 \\
(0.56-1.57)\end{array}$ \\
\hline Clinical improvement after 21 days, $n(\%)$ & $53(57.6)$ & $117(60.6)$ & 0.70 & $\begin{array}{c}0.88 \\
(0.53-1.46)\end{array}$ \\
\hline Clinical improvement after 28 days, $n(\%)$ & $68(73.9)$ & $128(66.3)$ & 0.22 & $\begin{array}{c}1.44 \\
(0.83-2.50)\end{array}$ \\
\hline
\end{tabular}

Bold: statistical significance.

To improve the predictive value, a combination of several measures was also analyzed. As shown in Table 4, the best effectiveness of TOC administration can be achieved in patients with serum IL- $6>100 \mathrm{pg} / \mathrm{mL}$ and either $\mathrm{SpO} 2 \leq 90 \%$ or requiring normal or highflow oxygen supplementation. Statistically significant effectiveness was achieved regarding the risk of death, the need for mechanical ventilation, as well as clinical improvement after 21 and 28 days (Table 4). Significantly better survival among such patients treated with TOC was also demonstrated with a Kaplan-Meier analysis (Figure 2). 


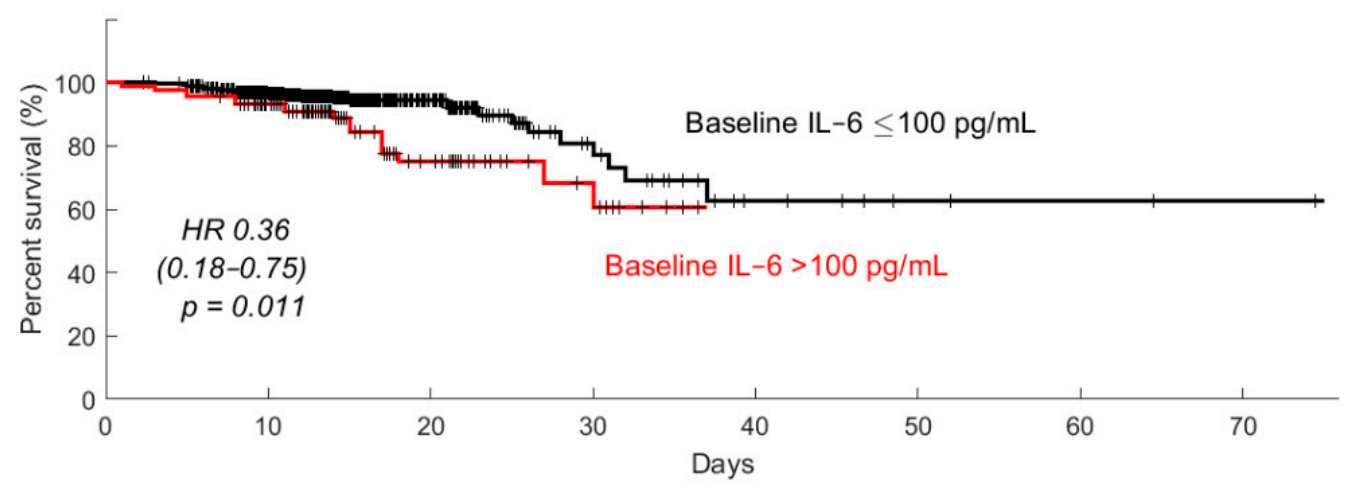

Baseline IL-6 $\leq 100 \mathrm{pg} / \mathrm{mL}$
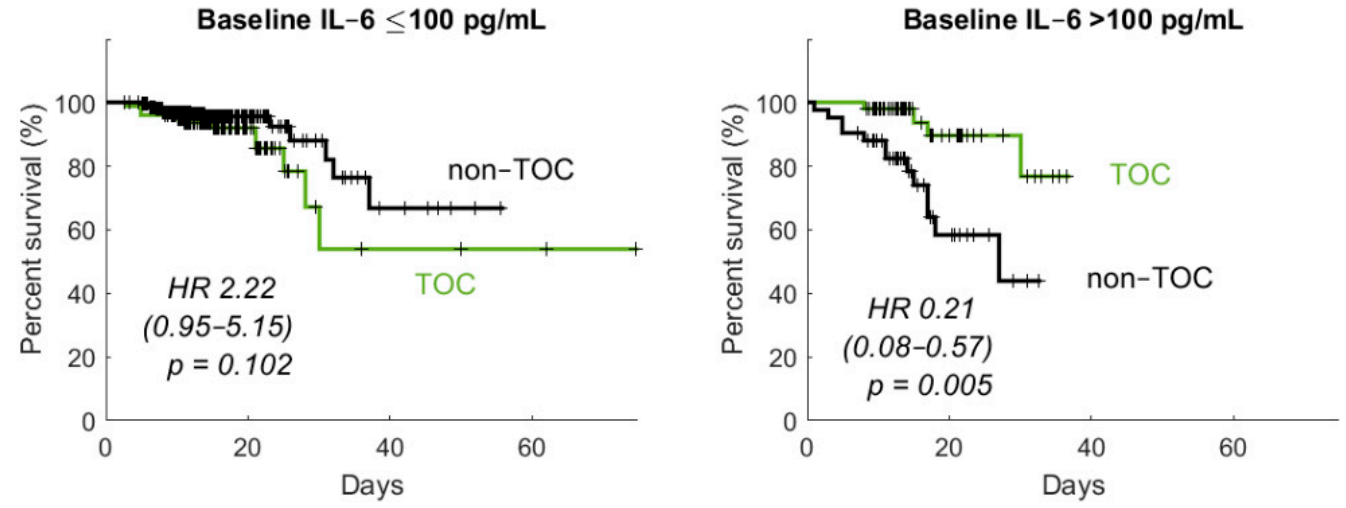

Figure 1. Kaplan-Meier graphs demonstrating the effect of tocilizumab versus no tocilizumab administration on patients' survival depending on the obtained optimal threshold baseline serum concentration of interleukin 6 . The hazard ratios (HR) and their $95 \%$ confidence intervals are provided as well as the log-rank test $\mathrm{p}$-values.

Table 3. Correlations between baseline serum IL-6 vs. selected clinical and laboratory indices.

\begin{tabular}{ccccccc}
\hline IL-6 Versus & \multicolumn{2}{c}{$\begin{array}{c}\text { All Patients } \\
(\boldsymbol{n}=\mathbf{8 2 5})\end{array}$} & \multicolumn{2}{c}{$\begin{array}{c}\text { SpO2 } \leq \mathbf{9 0} \% \\
(\boldsymbol{n}=\mathbf{3 7 2})\end{array}$} & \multicolumn{2}{c}{$\begin{array}{c}\text { SpO2 91-95\% } \\
(\boldsymbol{n}=\mathbf{4 5 3})\end{array}$} \\
\hline Age & $\mathbf{r}_{\mathbf{s}}$ & $\boldsymbol{p}$ & $\mathbf{r}_{\mathbf{s}}$ & $\boldsymbol{p}$ & $\mathbf{r}_{\mathbf{s}}$ & $\boldsymbol{p}$ \\
\hline BMI & 0.15 & $<0.001$ & 0.07 & 0.32 & 0.18 & 0.002 \\
\hline SpO ${ }_{2}$ & -0.01 & 0.86 & -0.03 & 0.63 & 0.01 & 0.89 \\
\hline CRP & 0.58 & $<0.001$ & 0.44 & $<0.001$ & 0.68 & $<0.001$ \\
\hline Procalcitonin & 0.40 & $<0.001$ & 0.37 & $<0.001$ & 0.36 & $<0.001$ \\
\hline WBC & 0.26 & $<0.001$ & 0.22 & $<0.001$ & 0.25 & $<0.001$ \\
\hline Lymphocytes & -0.21 & $<0.001$ & -0.05 & 0.42 & -0.28 & $<0.001$ \\
\hline Neutrophils & 0.32 & $<0.001$ & 0.23 & $<0.001$ & 0.37 & $<0.001$ \\
\hline Platelets & -0.10 & 0.04 & -0.10 & 0.14 & -0.13 & 0.03 \\
\hline D-dimers & 0.28 & $<0.001$ & 0.20 & 0.003 & 0.27 & $<0.001$ \\
\hline ALT & 0.13 & 0.03 & 0.10 & 0.13 & 0.10 & 0.09 \\
\hline
\end{tabular}


Table 4. Tocilizumab effect on rates of death, need for mechanical ventilation, and clinical improvement depending on combinations of possible outcomes predictors.

$\begin{array}{llllc}\text { Outcomes } & \text { Tocilizumab } & \text { No Tocilizumab } & p & \text { OR } \\ (95 \% \mathrm{CI})\end{array}$

Baseline IL6 $>100 \mathrm{pg} / \mathrm{mL}$ and requiring normal or high-flow oxygen supplementation (5 or 6 scores in ordinal scale)

\begin{tabular}{|c|c|c|c|c|}
\hline$n$ & 53 & 34 & & \\
\hline Death, $n(\%)$ & $8(15.1)$ & $13(38.2)$ & 0.02 & $\begin{array}{c}0.18 \\
(0.06-0.52)\end{array}$ \\
\hline Mechanical ventillation, $n(\%)$ & $8(15.1)$ & $7(20.6)$ & 0.57 & $\begin{array}{c}0.68 \\
(0.22-2.10)\end{array}$ \\
\hline Clinical improvement after 14 days, $n(\%)$ & $20(37.7)$ & $10(29.4)$ & 0.49 & $\begin{array}{c}1.45 \\
(0.58-3.66)\end{array}$ \\
\hline Clinical improvement after 21 days, $n(\%)$ & $33(62.3)$ & $13(38.2)$ & 0.047 & $\begin{array}{c}2.66 \\
(1.10-6.47)\end{array}$ \\
\hline Clinical improvement after 28 days, $n(\%)$ & $38(71.7)$ & $16(47.1)$ & 0.02 & $\begin{array}{c}2.85 \\
(1.16-7.01)\end{array}$ \\
\hline
\end{tabular}

\begin{tabular}{|c|c|c|c|c|}
\hline \multicolumn{5}{|c|}{ Baseline IL6 > $100 \mathrm{pg} / \mathrm{mL}$ and $\mathrm{SpO} 2<90 \%$} \\
\hline$n$ & 37 & 24 & & \\
\hline Death, $n(\%)$ & $4(10.8)$ & $12(50.0)$ & $<0.001$ & $\begin{array}{c}0.07 \\
(0.02-0.27)\end{array}$ \\
\hline Mechanical ventillation, $n(\%)$ & $2(5.4)$ & $7(29.2)$ & 0.02 & $\begin{array}{c}0.14 \\
(0.03-0.74)\end{array}$ \\
\hline Clinical improvement after 14 days, $n(\%)$ & $12(32.4)$ & $3(12.5)$ & 0.12 & $\begin{array}{c}3.36 \\
(0.83-13.52)\end{array}$ \\
\hline Clinical improvement after 21 days, $n(\%)$ & $24(64.9)$ & $6(25.0)$ & 0.004 & $\begin{array}{c}5.53 \\
(1.76-17.40) \\
\end{array}$ \\
\hline Clinical improvement after 28 days, $n(\%)$ & $28(75.7)$ & $9(37.5)$ & 0.004 & $\begin{array}{c}5.18 \\
(1.70-17.84)\end{array}$ \\
\hline \multicolumn{5}{|c|}{ Baseline IL6 > $100 \mathrm{pg} / \mathrm{mL}$ and CRP > $200 \mathrm{mg} / \mathrm{L}$} \\
\hline$n$ & 32 & 26 & & \\
\hline Death, $n(\%)$ & $5(15.6)$ & $7(26.9)$ & 0.34 & $\begin{array}{c}0.50 \\
(0.14-1.82)\end{array}$ \\
\hline Mechanical ventillation, $n(\%)$ & $1(3.1)$ & $4(15.4)$ & 0.16 & $\begin{array}{c}0.18 \\
(0.02-1.70)\end{array}$ \\
\hline Clinical improvement after 14 days, $n(\%)$ & $11(34.4)$ & $8(30.8)$ & 1.00 & $\begin{array}{c}1.18 \\
(0.39-3.57)\end{array}$ \\
\hline Clinical improvement after 21 days, $n(\%)$ & $18(56.3)$ & $15(57.7)$ & 1.00 & $\begin{array}{c}0.94 \\
(0.33-2.68)\end{array}$ \\
\hline Clinical improvement after 28 days, $n(\%)$ & $24(75.0)$ & $18(69.2)$ & 0.77 & $\begin{array}{c}1.33 \\
(0.42-4.23)\end{array}$ \\
\hline \multicolumn{5}{|c|}{ Baseline IL6 > $100 \mathrm{pg} / \mathrm{mL}$ and CRP $>200 \mathrm{mg} / \mathrm{L}$ and $\mathrm{SpO} 2<90 \%$} \\
\hline$n$ & 21 & 13 & & \\
\hline Death, $n(\%)$ & $4(19.0)$ & $5(38.5)$ & 0.26 & $\begin{array}{c}0.37 \\
(0.08-1.80)\end{array}$ \\
\hline Mechanical ventillation, $n(\%)$ & $1(4.8)$ & $3(23.1)$ & 0.27 & $\begin{array}{c}0.17 \\
(0.01-1.81)\end{array}$ \\
\hline Clinical improvement after 14 days, $n(\%)$ & $6(28.6)$ & $3(23.1)$ & 1.00 & $\begin{array}{c}1.33 \\
(0.27-6.61)\end{array}$ \\
\hline Clinical improvement after 21 days, $n(\%)$ & $11(52.4)$ & $5(38.5)$ & 0.49 & $\begin{array}{c}1.76 \\
(0.43-7.19)\end{array}$ \\
\hline Clinical improvement after 28 days, $n(\%)$ & $15(71.4)$ & $8(61.5)$ & 0.71 & $\begin{array}{c}1.56 \\
(0.36-6.76)\end{array}$ \\
\hline
\end{tabular}


Baseline IL- $6>100 \mathrm{pg} / \mathrm{mL}$ and requiring normal or high-flow oxygen supplementation

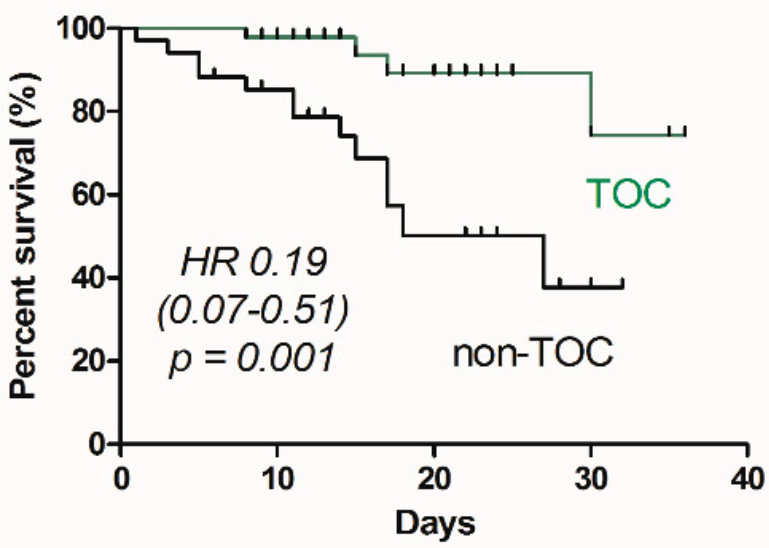

Baseline IL-6 $>100 \mathrm{pg} / \mathrm{mL}$ and baseline $\mathrm{SpO} 2<90 \%$

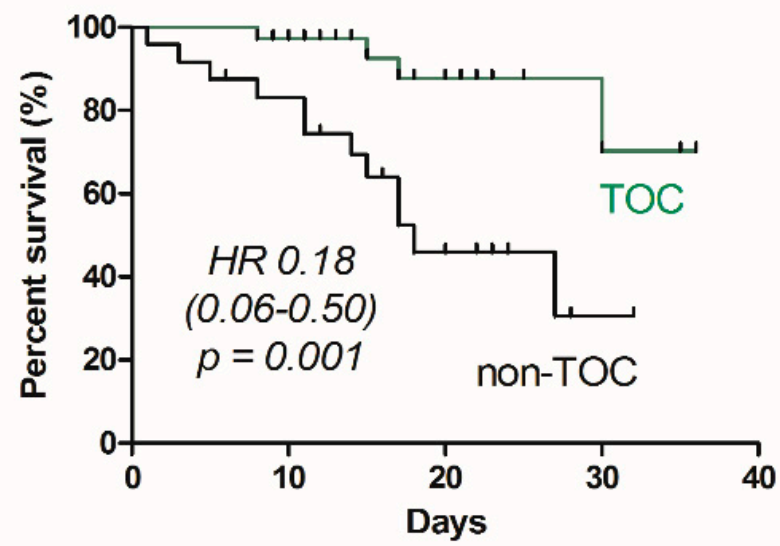

Figure 2. Kaplan-Meier graphs demonstrating the effect of tocilizumab versus no tocilizumab administration on patients' survival depending on baseline serum concentration of interleukin 6 . The hazard ratios (HR) and their 95\% confidence intervals are provided as well as the log-rank test $p$-values.

TOC

Adverse events related to therapy were infrequent and reported in $21.7 \%$ and $17.3 \%$ of patients in TOC and non-TOC arms, respectively. As shown in Table 5, the most frequent was an elevation of ALT activities, diarrhea, and prolonged QT interval. Prevalence of adverse events was similar in both arms (Table 5). No secondary infections were noticed in patients treated with TOC.

Table 5. Prevalence of adverse events.

\begin{tabular}{cccc}
\hline Adverse Events & Tocilizumab & No Tocilizumab & $p$ \\
\hline $\boldsymbol{n}$ & 170 & 655 & \\
\hline ALT elevation, $n(\%)$ & $17(10.0)$ & $40(6.1)$ & 0.09 \\
\hline Diarrhea, $n(\%)$ & $8(4.7)$ & $39(5.9)$ & 0.71 \\
\hline Prolonged QT interval, $n(\%)$ & $3(1.8)$ & $11(1.7)$ & 1.00 \\
\hline Nausea, $n(\%)$ & $2(1.2)$ & $12(1.8)$ & 0.75 \\
\hline Other, $n(\%)$ & $7(4.7)$ & $11(1.7)$ & 0.07 \\
\hline All adverse events, $n(\%)$ & $37(21.7)$ & $113(17.3)$ & 0.18 \\
\hline
\end{tabular}

\section{Discussion}

Uncontrolled immune activation with high-level release of various pro-inflammatory cytokines is a hallmark of not only the lung damage but also multiorgan damage during later phases of COVID-19. It is usually termed as a "cytokine storm" and observed mainly during the second and third week of symptomatic disease in patients with severely impaired oxygen saturation [18]. Unsurprisingly, anti-cytokine agents including anti-IL-1R and anti-IL6R antagonists were among important candidates in the therapy of later stages of COVID-19. One of the rationales was a good suppressive effect of tocilizumab in cytokine release syndrome during CAR T-cells therapy [19]. Regardless of the pathophysiological link, various randomized and observational studies of TOC, while suggesting some benefit, did not bring clear evidence supporting its use in COVID-19. This may reflect the unique features of individual immune responses to pathogens, as well as the necessity of a complex and personalized approach in prescribing and timing immunomodulatory treatment. 
Proposing clinical trial protocol taking such diversity into account proves to be challenging; thus, personalized medicine relies on observational research and real-life experiences.

Results of our real-world evidence study could not only potentially explain the lack of effect of TOC observed in other studies but also provide information on the optimal use of this agent. In our cohort, similar to the first two randomized controlled trials (RCT), TOC did not decrease overall mortality in hospitalized patients with COVID-19. In the first study by Stone JH et al. [14], 83\% of 243 COVID-19 subjects requiring oxygen supplementation but not mechanical ventilation were randomized to TOC $(8 \mathrm{mg} / \mathrm{kg}$, single dose) or placebo. In this study, the hazard ratio (HR) for death was 0.83 , which was not significant, with broad $95 \%$ confidence intervals ( 0.38 to $1.81, p=0.64$ ) suggesting heterogeneity of effect probably depending on other clinical variables not found in the publication. In another RCT by Hermine et al. [12] including 131 patients with COVID-19 pneumonia requiring oxygen supplementation but not mechanically ventilated, 64 were randomized to TOC $(8 \mathrm{mg} / \mathrm{kg}$, twice) or placebo. Likewise, the adjusted HR for 28-day mortality was 0.92 (90\%CI 0.33-2.53). On the other hand, on day 14 in TOC-group, $12 \%$ fewer patients needed non-invasive or mechanical ventilation, or $12 \%$ less died (HR 0.58 ; $90 \%$ CI $0.33-1.00$ ). Only the most recent and largest RCT by Salama et al. [13] showed a survival benefit in patients treated with TOC. In this study, 377 subjects with COVID-19 pneumonia, $64 \%$ requiring lowflow and $26 \%$ on non-invasive and high flow oxygen supplementation, were randomized to TOC ( $\mathrm{n}=259,8 \mathrm{mg} / \mathrm{kg}$, one or two doses) or placebo. By day 28 , HR for mechanical ventilation or death was 0.56 (95\%CI: $0.33-0.97, p=0.04$ ), while still the number of patients who died by that day of any reason was $10.4 \%$ in TOC group vs. $8.6 \%$ in the placebo group.

Summarizing all aforementioned randomized clinical trials despite a rather homogenous population included, which is patients with COVID-19 pneumonia mainly requiring oxygen supplementation but not ventilated, it was not possible to visualize the obvious survival benefit of TOC. Similarly in our study, the overall mortality was comparable between TOC and non-TOC patients (odds ratio, OR 1.05; 95\%CI: 0.61-1.80). Furthermore, the group receiving TOC showed even lesser odds of clinical improvement after 14 and 21 days of therapy (OR 0.61 and 0.64, respectively), while it was comparable to the standard-of-care therapy at the end of observation, i.e., after 28 days (OR 0.87). On the other hand, only analyses in specific subgroups showed not only a survival benefit but also a more rapid clinical improvement in patients treated with TOC. It was quite striking that in previous studies, mortality HR had quite outsized confidence intervals, suggesting another factor playing the predictive role of TOC efficacy. Surprisingly enough, cited above RCT did not evaluate baseline IL-6 serum concentration even when TOC is aimed at blocking an IL-6 proinflammatory pathway. Indeed, we performed a detailed subgroup analysis aiming at the development of predictors of TOC response in COVID-19 subjects. Not unpredictably, the best response to TOC concerning decreasing 28-day mortality (OR $=0.27 ; 95 \% \mathrm{CI}$ : $0.10-0.78,11 \%$ vs. $31 \%, p=0.02)$ was observed in subjects with baseline serum IL-6 $>100 \mathrm{pg} / \mathrm{mL}$, while it was not observed in subject with baseline IL-6 $50-100 \mathrm{pg} / \mathrm{mL}$ and below $50 \mathrm{pg} / \mathrm{mL}$. Importantly, an IL-6 level of more than $100 \mathrm{pg} / \mathrm{mL}$ observed in approximately $18 \%$ of all studied patients possibly explain why the difference was not noted in overall studied groups in the aforementioned RCT but also in our study. This observation also underlines the pathogenetic complexity of cytokine imbalance during COVID-19. It is known that cytokine storm in COVID-19 consists of various, not necessarily overlapping, soluble immune mediators (SIMs) including IL-1 $\beta$, IL-6, IL-8, and tumor necrosis factor-alpha (TNF- $\alpha$ ) which could yield different predictive value [20]. Interestingly, Mathew et al. [21] in their elegant study had shown at least three different immunotypes of COVID-19, 1-3, depending on the cluster of differentiation of (CD)4+ cell, CD8+ cell, and B-cell, and plasmablasts activation/exhaustion, which was associated with different outcomes but also most likely with different levels of cytokines. Interestingly, despite baseline IL-6, the effect of TOC in our study did not depend on baseline CRP, D-dimer, or lymphocyte concentrations, which are also regarded as factors associated with prognosis [22]. 
Another important finding of our study was that the highest reduction in mortality, the need for mechanical ventilation, and best clinical improvement at day 28 in patients receiving TOC vs. standard-of-care (SOC) therapy was observed in patients with baseline IL-6 $>100 \mathrm{pg} / \mathrm{mL}$ and $\mathrm{SpO} 2<90 \%$ (11 vs. $50 \%, 5$ vs. $29 \%$, and 75 vs. $37 \%$, respectively), which was not the case in subjects with $\mathrm{SpO} 2 \geq 90 \%$. This observation might further underline that in subjects with severe hypoxia, further deregulation between IL-6 levels and other cytokines is present and possibly IL-6 activation is deeper and not counterbalanced by regulatory mechanism, which could explain why the effect of TOC is more significant. In addition, in our study, correlation analyses showed the correlation pattern of IL-6 and some soluble immune mediators are different in patients with oxygen saturation lower and higher/equal to $90 \%$.

The results of our study should be taken with some caution because of its retrospective real-world evidence design and because of the smaller number of participants in some subgroup analyses. Moreover, some patients in both arms received additional medication, which could affect the outcome of the disease. On the other hand, patients receiving TOC and non-TOC SOC therapies seem to be well balanced with regard to comorbidities and co-medications for COVID-19, and undoubtedly its advantage is the assessment of baseline serum IL-6 concentration. Indeed, while only one other single-center study showed a beneficial effect of TOC mainly in patients with higher IL-6 [23], our data in a real-world large dataset seems to guide the effective use of TOC in COVID-19. Safety profile of TOC in our study was good. Adverse events were infrequent and mild. However, risk of secondary infections should always be considered [24].

\section{Conclusions}

In conclusion, the possible benefit from the treatment of COVID-19 with tocilizumab can be achieved in selected subpopulations only. This regimen can reduce mortality and the need for mechanical ventilation in patients with a baseline concentration of interleukin 6 exceeding $100 \mathrm{pg} / \mathrm{mL}$, particularly if they need oxygen supplementation due to oxygen saturation of $\leq 90 \%$. Patients who worsened within the initial 7 days of hospitalization can also obtain some benefits from tocilizumab administration, but it should be clarified in further studies on a larger number of patients.

Author Contributions: R.F.-designed research, performed research, analyzed data, wrote majority of the article, performed the final submission. J.J. and J.P.- - analyzed data, performed statistics, drawed figures, and wrote some parts of the article. D.Z.-M.-collected data, analyzed data, and wrote some parts of the article. M.R. (Magdalena Rogalska), T.Ł., A.B.-K., B.B., M.T.-Z., K.K., M.R. (Marta Rorat), P.L., D.K., J.K., P.P., A.P., I.M.-L., K.T., M.P., K.S.—collected data and analyzed data. All authors have read and agreed to the published version of the manuscript.

Funding: This research was funded by Medical Research Agency, grant number 2020/ABM/COVID19/ PTEILCHZ and Polish Association of Epidemiologists and Infectiologists.

Institutional Review Board Statement: The study was conducted according to the guidelines of the Declaration of Helsinki, and approved by the Ethics Committee of Medical University of Białystok (29 October 2020, number APK.002.303.2020).

Informed Consent Statement: Informed consent was obtained from all subjects involved in the study.

Data Availability Statement: Data supporting reported results can be provided upon request from the corresponding author. 
Conflicts of Interest: R.F. reports grants from Abbvie, Gilead, and Merck; personal fees from Gilead, Abbvie, Merck, and Roche; and non-financial support from Abbvie, Gilead, and Merck outside the submitted work. D.Z.M. and P.P. reports personal fees from Gilead and Abbvie, outside the submitted work. JJ reports personal fees from Gilead, Abbvie, Bausch Health, Merck, Promed, and Roche, and non-financial support from Abbvie, Gilead, and Merck outside the submitted work. K.S. reports personal fees from Gilead, Abbvie, and Merck, outside the submitted work. K.T. reports personal fees from Gilead, Abbvie, Merck, Promed, and Roche, and non-financial support from Abbvie, Gilead, and Merck outside the submitted work. J.K. reports personal fees from Gilead, Merck, ViiV, and Janssen outside the submitted work. IML reports personal fees from Gilead, Abbvie, and Pfizer. A.B.K., J.P., B.B., K.K., M.P., A.P., D.K., M.T.Z., C.I., MRog, and MRor declare no competing interests.

\section{References}

1. Mulangu, S.; Dodd, L.E.; Davey, R.T., Jr.; Mbaya, O.T.; Proschan, M.; Mukadi, D.; Manzo, M.L.; Nzolo, D.; Oloma, A.T.; Ibanda, A.; et al. A Randomized, Controlled Trial of Ebola Virus Disease Therapeutics. N. Engl. J. Med. 2019, 381, 2293-2303. [CrossRef]

2. Sheahan, T.P.; Sims, A.C.; Graham, R.L.; Menachery, V.D.; Gralinski, L.E.; Case, J.B.; Leist, S.R.; Krzysztof, P.; Feng, J.Y.; Trantcheva, I.; et al. Broad-spectrum antiviral GS-5734 inhibits both epidemic and zoonotic coronaviruses. Sci. Transl. Med. 2017, 9, eaal3653. [CrossRef]

3. Beigel, J.H.; Tomashek, K.M.; Dodd, L.E.; Mehta, A.K.; Zingman, B.S.; Kalil, A.C.; Hohmann, E.; Chu, H.Y.; Luetkemeyer, A.; Kline, S.; et al. Remdesivir for the Treatment of Covid-19_Preliminary Report. N. Engl. J. Med. 2020, 383, 1813-1826. [CrossRef]

4. Goldman, J.D.; Lye, D.C.; Hui, D.S.; Marks, K.M.; Bruno, R.; Montejano, R.; Spinner, C.D.; Galli, M.; Ahn, M.-Y.; Nahass, R.G.; et al. Remdesivir for 5 or 10 Days in Patients with Severe Covid-19. N. Engl. J. Med. 2020, 83, 1827-1837. [CrossRef]

5. Flisiak, R.; Zarębska-Michaluk, D.; Berkan-Kawińska, A.; Tudrujek-Zdunek, M.; Rogalska, M.; Piekarska, A.; Kozielewicz, D.; Kłos, K.; Rorat, M.; Bolewska, B.; et al. Remdesivir-based therapy improved recovery of patients with COVID-19 in the SARSTer multicentre, real-world study. Pol. Arch. Intern. Med. 2021, 131, 103-110.

6. Tang, N.; Bai, H.; Chen, X.; Gong, J.; Li, D.; Sun, Z. Anticoagulant treatment is associated with decreased mortality in severe coronavirus disease 2019 patients with coagulopathy. J. Thromb. Haemost. 2020, 18, 1094-1099. [CrossRef] [PubMed]

7. Horby, P.; Lim, W.S.; Emberson, J.R.; Mafham, M.; Bell, J.L.; Linsell, L.; Staplin, N.; Brightling, C.; Ustianowski, A.; Elmahi, E.; et al. Dexamethasone in hospitalized patients with Covid-19_preliminary report. N. Engl. J. Med. 2021, 384, 693-704. [PubMed]

8. Jose, R.J.; Manuel, A. COVID-19 cytokine storm: The interplay between inflammation and coagulation. Lancet Respir. Med. 2020, 8, e46-e47. [CrossRef]

9. Tomasiewicz, K.; Piekarska, A.; Stempkowska-Rejek, J.; Serafińska, S.; Gawkowska, A.; Parczewski, M.; Niścigorska-Olsen, J.; Łapiński, T.W.; Zaręska-Michaluk, D.; Kowalska, J.D.; et al. Tocilizumab for patients with severe COVID-19: A retrospective, multi-center study. Expert Rev. AntiInfect. Ther. 2021, 19, 93-100. [CrossRef] [PubMed]

10. Liu, B.; Li, M.; Zhou, Z.; Guan, X.; Xiang, Y. Can we use interleukin-6 (IL-6) blockade for coronavirus disease 2019 (COVID-19)induced cytokine release syndrome (CRS)? J. Autoimmun. 2020, 111, 102452. [CrossRef]

11. Rosas, I.O.; Bräu, N.; Waters, M.; Go, R.C.; Hunter, B.D.; Bhagani, S.; Skiest, D.; Aziz, M.S.; Cooper, N.; Douglas, I.S.; et al. Tocilizumab in hospitalized patients with severe COVID-19 pneumonia. N. Engl. J. Med. 2021. [CrossRef]

12. Hermine, O.; Mariette, X.; Tharaux, P.L.; Resche-Rigon, M.; Porcher, R.; Ravaud, P. Effect of Tocilizumab vs Usual Care in Adults Hospitalized With COVID-19 and Moderate or Severe Pneumonia: A Randomized Clinical Trial. JAMA Intern. Med. 2021, 181, 32-40. [CrossRef]

13. Salama, C.; Han, J.; Yau, L.; Reiss, W.G.; Kramer, B.; Neidhart, J.D.; Criner, G.J.; Kaplan-Lewis, E.; Baden, R.; Pandit, L.; et al. Tocilizumab in Patients Hospitalized with Covid-19 Pneumonia. N. Engl. J. Med. 2021, 384, 20-30. [CrossRef]

14. Stone, J.H.; Frigault, M.J.; Serling-Boyd, N.J.; Fernandes, A.D.; Harvey, L.; Foulkes, A.S.; Horick, N.K.; Healy, B.C.; Shah, R.; Bensaci, A.M.; et al. Efficacy of Tocilizumab in Patients Hospitalized with Covid-19. N. Engl. J. Med. 2020, 383, 2333-2344. [CrossRef]

15. Flisiak, R.; Horban, A.; Jaroszewicz, J.; Kozielewicz, D.; Pawłowska, M.; Parczewski, M.; Piekarska, A.; Simon, K.; Tomasiewicz, K.; Zarębska-Michaluk, D. Management of SARS-CoV-2 infection: Recommendations of the Polish Association of Epidemiologists and Infectiologists as of March 31, 2020. Pol. Arch. Intern. Med. 2020, 130, 352-357. [PubMed]

16. Flisiak, R.; Horban, A.; Jaroszewicz, J.; Kozielewicz, D.; Pawlowska, M.; Parczewski, M.; Piekarska, A.; Simon, K.; Tomasiewicz, K.; Zarebska-Michaluk, D. Management of SARS-CoV-2 infection: Recommendations of the Polish Association of Epidemiologists and Infectiologists. Annex no. 1 as of June 8, 2020. Pol. Arch. Intern. Med. 2020, 130, 557-558.

17. Flisiak, R.; Parczewski, M.; Horban, A.; Jaroszewicz, J.; Kozielewicz, D.; Pawłowska, M.; Piekarska, A.; Simon, K.; Tomasiewicz, K.; Zaręsska-Michaluk, D. Management of SARS-CoV-2 infection: Recommendations of the Polish Association of Epidemiologists and Infectiologists. Annex no. 2 as of October 13, 2020. Pol. Arch. Intern. Med. 2020, 130, 915-918. [CrossRef] [PubMed]

18. Fajgenbaum, D.C.; June, C.H. Cytokine Storm. N. Engl. J. Med. 2020, 383, 2255-2273. [CrossRef] [PubMed]

19. Le, R.Q.; Li, L.; Yuan, W.; Shord, S.S.; Nie, L.; Habtemariam, B.A.; Przepiorka, D.; Farrell, A.T.; Pazdur, R. FDA Approval Summary: Tocilizumab for Treatment of Chimeric Antigen Receptor T Cell-Induced Severe or Life-Threatening Cytokine Release Syndrome. Oncologist 2018, 23, 943-947. [CrossRef] 
20. Del Valle, D.M.; Kim-Schulze, S.; Huang, H.H.; Beckmann, N.D.; Nirenberg, S.; Wang, B.; Lavin, Y.; Swartz, T.H.; Madduri, D.; Stock, A.; et al. An inflammatory cytokine signature predicts COVID-19 severity and survival. Nat. Med. 2020, 26, 1636-1643. [CrossRef]

21. Mathew, D.; Giles, J.R.; Baxter, A.E.; Oldridge, D.A.; Greenplate, A.R.; Wu, J.E.; Alanio, C.; Kuri-Cervantes, L.; Pampena, M.B.; D'Andrea, K.; et al. Deep immune profiling of COVID-19 patients reveals distinct immunotypes with therapeutic implications. Science 2020, 369, eabc8511. [CrossRef]

22. Mueller, A.A.; Tamura, T.; Crowley, C.P.; DeGrado, J.R.; Haider, H.; Jezmir, J.L.; Keras, G.; Penn, E.H.; Massaro, A.F.; Kim, E.Y. Inflammatory biomarker trends predict respiratory decline in COVID-19 patients. Cell Rep. Med. 2020, 1, 100144. [CrossRef] [PubMed]

23. Galván-Román, J.M.; Rodríguez-García, S.C.; Roy-Vallejo, E.; Marcos-Jiménez, A.; Sánchez-Alonso, S.; Fernández-Díaz, C.; Alcaraz-Serna, A.; Mateu-Albero, T.; Rodríguez-Cortes, P.; Montes, N.; et al. IL-6 serum levels predict severity and response to tocilizumab in COVID-19: An observational study. J. Allergy Clin. Immunol. 2020, 147, 72-80. [CrossRef] [PubMed]

24. Deana, C.; Vetrugno, L.; Bassi, F.; De Monte, A. Tocilizumab administration in COVID-19 patients: Water on the fire or gasoline? Med. Mycol. Case Rep. 2021, 31, 32-34. [CrossRef] [PubMed] 